\title{
Research on the Construction of Characteristic Database of University Library
}

\author{
Yanhui Zhang ${ }^{1}$, Xiaoyue $\mathbf{Y v}^{2}$ \\ ${ }^{1}$ Jilin University, Changchun, Jilin, 130021 \\ ${ }^{2}$ University Changchun, Jilin, 130021
}

Keywords: university library, database, construction and application

\begin{abstract}
Aiming at the current situation of the construction of the characteristic database of university libraries, combined with the characteristics of the era of big data, this paper puts forward the idea of constructing the characteristic data of university libraries under the big data environment, from data label, sub-node construction, virtual large database system and user access mechanism, the construction mode, implementation mode and user access mechanism of the featured large database system. The "data tags" is used to virtualize the different types of characteristic databases scattered in different colleges and universities to achieve centralized display, unified retrieval and co-construction and sharing of featured data.
\end{abstract}

\section{Introduction}

With the advent of the information age, digital information, as a form of resources, plays an increasingly important role in the construction and development of university libraries. It has become an important symbol to measure the construction of knowledge information resources in a university, and has increasingly become a university library. The core and key to the implementation of digital library construction. However, university libraries should establish a library of document information resources with their own characteristics according to their professional advantages and regional development requirements, especially through the digital processing of library special collections and the collection and arrangement of information resources with colleges and regions. Digital processing, to strengthen the construction of university library characteristic database, to achieve resource application and sharing; and to provide users with unique services to maximize the learning and research needs of the user, for education, personnel training and social and economic development services. For example, Sichuan University has built the Bashu Culture Database, and Hunan University has built the Hunan Character Database, which has become an effective carrier for disseminating local culture and promoting school resource sharing. The characteristic database of university libraries under the perspective of building knowledge management is the inevitable response of university libraries to the development of information age. To cope with this change, universities should comprehensively implement funds investment, software and hardware construction, management talents and scientific management mechanisms. In order to realize the construction of the characteristic database of university libraries, an effective path is opened.

\section{The status quo of the construction of university characteristic database}

The construction of characteristic database of colleges and universities in China began in the 1990s. Article 11 of the third chapter of the Regulations of the General Higher Education Library revised by the Ministry of Education in 2002 states: "The library of higher education institutions shall be based on the needs of school teaching and scientific research, and according to the characteristics of the collection and the regional or systematic literature support system and construction. The division of labor, the development of characteristic digital resources and the construction of virtual resources on the Internet, the integration of physical resources and virtual resources, the formation of a unified online collection system." In 2003, the Ministry of Education developed the "General College Books" on the basis of the "Procedures" Library Evaluation Index 
System (Consultation Draft), "System" will be used as an important indicator to evaluate the construction of university literature resources. In July 1999, the China Higher Education Document Security System (CALIS) began to build a special library project. By the end of 2011, the CALIS featured database construction was funded in the first and second phases to build 83 characteristics with Chinese characteristics, local characteristics, and higher education. Resource characteristics, service literature and scientific research and economic construction, convenient and practical, advanced technology literature database. China's 211 Engineering University Library Since the late 1990s, there have been 263 different types of featured databases in 64211 engineering university libraries. Judging from the characteristic database of the established university library, its types are rich in subject matter and diverse in form. According to the database storage description method, it can be divided into full-text type, numerical type, fact-based source database and catalogue bibliographic reference database such as bibliography, bibliography and abstracts; according to the document content stored in the characteristic library, there are: collection characteristic database, For example, the "College of Ancient Literature Resource Library" of Peking University Library and the "China Shipping Information Resource Library" of Dalian Maritime University Library; Subject characteristic database, such as the "Mining Engineering Database" of China University of Mining and Technology Library, "Road and Engineering Database" of Changsha University of Science and Technology Library under construction; 3 School characteristic database, such as "Xi'an Jiaotong University Library" of Xi'an Jiaotong University Library, Zhejiang University Library's "Zheda Library", Zhongshan University "Sun Yat-sen Digital Library" of the library; local characteristic databases, such as the "Yangtze River Resources Database" of the Wuhan University Library, and the "Great Pearl River Delta Literature Database" of the Sun Yat-sen University Library; Other characteristic databases According to the way of building the database, there are self-built databases and imported special databases. As an important part of university collections, the featured database has made great achievements for the personalized and characteristic service of colleges and universities, and has received very good results. However, on the whole, the construction of the characteristic database of colleges and universities is still in its infancy, and there is no perfect construction system, sharing system and service system.

\section{Problems in the construction of university characteristic database}

Throughout the construction and service of the characteristic database of colleges and universities in China, although there have been some achievements, there are still many problems, mainly as follows: 1 The database is small in scale, lacks overall planning, can not find the direction and focus of the database, and can not find 2 characteristics; 2 lack of unified digital processing, resource description, resource organization, resource interoperability and resource services standards and norms, affecting the compatibility, interoperability and sustainability of the featured database, thus limiting its Sharing. The survey found that the featured libraries funded by the CALIS Regional Center have uniform standard requirements, but the characteristic database that has not been constructed by the CALIS regional center organization has not met the corresponding standard for building the database; 3 The software platform is not hierarchical, affecting the characteristic database. Scalability, ease of use, and sharing. Among the featured databases that have been built, most colleges and universities purchase mature commercial software certified by CALIS Center, but some colleges and universities use software platforms developed independently or jointly for financial problems; 4 lack of recognition for featured databases The quality tracking and evaluation system has caused enthusiasm in the early stage of building a database. After the completion of the database construction, it will not be updated and maintained, and it will not be promoted or promoted. It is contrary to the original intention of building a characteristic library. 5 Most of the self-built characteristic databases of colleges and universities are limited to the domain network or The use of the local area network, the external network can not be consulted: Some researchers visited the 68 featured databases built by the CALIS Center through the education network and the telecommunication network at different times, and found that only 18 were accessible, accounting for only $26.5 \%$ of the total number of featured databases. There is no real 
sharing.

\section{University Library's Characteristic Collection Database Construction Strategy}

For the library, it is very difficult to create a special database by relying solely on the digital resource construction funds of the library. Therefore, the work of building a featured database can be upgraded to a higher level and supported by special funds. For example, the university library can incorporate the construction of the characteristic database into the school's annual work plan through the proposal of the representative conference, so that not only can the construction fund be guaranteed, but also the school can be more easily acquired during the construction of the characteristic database. Collaboration and support from various departments. In addition, more channels can be expanded to secure special funds. For example, through the application of provincial and municipal research projects, special research funding will be obtained; and other libraries with relevant database construction needs will be built and shared.

The creation of the featured database should be carried out in stages and a good construction plan should be drawn up. First of all, we must start from the most distinctive and most accumulated disciplines in the museum, and we must highlight the "features." The advantages of this are as follows: First, the resources of the most distinctive disciplines will be relatively rich, so that when building a characteristic database, it is relatively easy to produce results in data collection, and can be better promoted as a template to more disciplines. Second, according to the characteristic database built by the specialty, after the completion of the construction, its resource utilization rate will be relatively high, and it can maximize its role. The library can focus on building one or two specialized databasees. After accumulating certain resources and experience, the library will gradually increase the disciplines involved, that is, "first make the fine, then seek the whole".

The creation of a featured database is an arduous, long-term job that can be accomplished without relying on the power of three or two people. For the library, it is not realistic to deploy a special database for the staff. Therefore, the librarian-centered database construction mode can be transformed into a reader-centered database construction model, so that readers can participate in the characteristic database. Under construction, the librarian becomes a role of assisting counseling. For example, when a university library constructs a characteristic database, the teachers of the specialties involved in the database can be included in the database construction team. The professional teachers collect resources according to the subject content of the featured database, and guide and control the screening and selection of resources. In addition, some senior students with relatively good computer skills can be recruited into the construction team of the featured database to assist them in resource collection, processing and entry. Librarians are mainly responsible for resource review and personnel organization. According to this model, a team of professionals with relevant professional knowledge background and knowledge of library science and computer skills will be established to truly "build and share", ensure the quality of the featured database, and avoid the knowledge of librarians. The structure is unreasonable and thus affects the construction of the database.

The main purpose of the construction of the characteristic database of university libraries is to provide services for teaching and research and student learning, without commercial purposes. However, there may still be infringement issues involved in improper handling. We should make full use of the "reasonable use" principle, "authorized use" and advanced technology protection to solve the intellectual property problem in database construction. On the one hand, database construction is the result of the hard work and wisdom of the participants. In the process of construction, a large amount of manpower, material resources, financial resources and technology must be invested. Such labor results must be protected by law and enjoy full intellectual property rights. On the other hand, Respect the author's labor results. For copyright-protected information materials, we can mark it when it is quoted, explain its source, and if necessary, it can also use some of the high-use, high-quality materials for paid downloading. For those who cannot obtain the right to use, Establish related links in the featured database to provide users with remote browsing and navigation. 


\section{Conclusion}

Although it is faced with the situation of "difficult to create and difficult to maintain", the creation of a database of library collections is still a very meaningful work. Because the library's characteristic collection database is established, it can not only meet the readers' needs for the use of the school's resources, but also help the related disciplines to establish a relatively standardized and unified teaching resource library, and also facilitate the promotion of cultural exchanges and digital archives of various professions. In one fell swoop. As long as the library continuously adjusts and improves from the sources of funding, resource content, staffing, etc., long-term adherence will definitely improve the construction level of the library's featured collection database and achieve remarkable results.

\section{References}

[1] Yang Zhirong. Investigation and Analysis of the Characteristic Resources Construction Network of the "211 Project" University Library in China [J]. Library Work and Study, 2012(7): 56-59.

[2] Wang Ping. Research on the Construction of Characteristic Resources of Higher Vocational College Libraries [J]. Jiangxi Library Journal, 2012(6):31-33.

[3] Liu Chenguang, et al. Wiki and its application in the library [J]. Journal of Medical Informatics, 2010 (3): 24-27.

[4] Zhang Aihong. Discussion on the Construction of Lingnan Music Characteristic Resource Database [J]. Library Research, 2013(1): 23-26.

[5] Chen Guogang. Building a Chinese Female Character Resource Library Based on Hdwiki[J]. Library Journal, 2010(8): 42-43. 